\title{
On discrete GB-splines
}

\author{
Boris I. Kvasov*
}

(Received 7 August 2000)

\begin{abstract}
Explicit formulae and recurrence relations are obtained for discrete generalized B-splines (discrete GB-splines for short). Properties of discrete GB-splines and their series are studied. It is shown that the series of discrete GB-splines is a variation diminishing function and the systems of discrete GB-splines are weak Chebyshev systems.
\end{abstract}

* School of Mathematics, Suranaree University of Technology, Nakhon Ratchasima 30000, ThaILAND. mailto: boris@math.sut.ac.th

${ }^{0}$ See http://anziamj . austms . org. au/V42/CTAC99/Kvas for this article and ancillary services, (c) Austral. Mathematical Soc. 2000. Published 27 Nov 2000. 


\section{Contents}

1 Introduction

C878

2 Discrete generalized splines

C879

3 Construction of discrete GB-splines

C882

4 Properties of discrete GB-splines

C885

5 Local approximation by discrete GB-splines

C887

6 Recurrence formulae for discrete GB-splines

C890

7 Series of discrete GB-splines (uniform case)

C892

References

C898

\section{Introduction}

The tools of generalized splines and GB-splines are widely used in solving problems of shape-preserving approximation (e.g., see [7]). Recently, in [1] a difference method for constructing shape-preserving hyperbolic tension splines as solutions of multipoint boundary value problems was developed. Such an approach permits us to avoid the computation of hyperbolic func- 
tions and has substantial other advantages. However, the extension of a mesh solution will be a discrete hyperbolic tension spline.

The contents of this paper is as follows. In Section 2 we give a definition of a discrete generalized spline. Next, we construct a minimum length local support basis (whose elements are denoted as discrete GB-splines) of the new spline; see Section 3. Properties of GB-splines are discussed in Section 4, while the local approximation by discrete GB-splines of a given continuous function from its samples is considered in Section 5. In Section 6 we derive recurrence formulae for calculations with discrete GB-splines. The properties of GB-spline series are summarized in Section 7.

\section{Discrete generalized splines}

Let a partition $\Delta: a=x_{0}<x_{1}<\cdots<x_{N}=b$ of the interval $[a, b]$ be given. We will denote by $S_{4}^{D G}$ the space of continuous functions whose restriction to a subinterval $\left[x_{i}, x_{i+1}\right], i=0, \ldots, N-1$ is spanned by the system of four linearly independent functions $\left\{1, x, \Phi_{i}, \Psi_{i}\right\}$. In addition, we assume that each function in $S_{4}^{D G}$ is smooth in the sense that for given $\tau_{i}^{L_{j}}>0$ and $\tau_{i}^{R_{j}}>0, j=i-1, i$, the values of its first and second central divided differences with respect to the points $x_{i}-\tau_{i}^{L_{i-1}}, x_{i}, x_{i}+\tau_{i}^{R_{i-1}}$ and $x_{i}-\tau_{i}^{L_{i}}$, $x_{i}, x_{i}+\tau_{i}^{R_{i}}$ coincide. 
Given a continuous function $S$ we introduce the difference operators

$$
\begin{aligned}
D_{1} S(x) \equiv D_{i, 1} S(x)= & \left(\lambda_{i}^{R_{i}} S\left[x-\tau_{i}^{L_{i}}, x\right]+\lambda_{i}^{L_{i}} S\left[x, x+\tau_{i}^{R_{i}}\right]\right)(1-t) \\
& +\left(\lambda_{i+1}^{R_{i}} S\left[x-\tau_{i+1}^{L_{i}}, x\right]+\lambda_{i+1}^{L_{i}} S\left[x, x+\tau_{i+1}^{R_{i}}\right]\right) t, \\
D_{2} S(x) \equiv D_{i, 2} S(x)= & 2 S\left[x-\tau_{i}^{L_{i}}, x, x+\tau_{i}^{R_{i}}\right](1-t) \\
& +2 S\left[x-\tau_{i+1}^{L_{i}}, x, x+\tau_{i+1}^{R_{i}}\right] t, \\
& x \in\left[x_{i}, x_{i+1}\right), \quad i=0, \ldots, N-1,
\end{aligned}
$$

where $\lambda_{j}^{R_{i}}=1-\lambda_{j}^{L_{i}}=\tau_{j}^{R_{i}} /\left(\tau_{j}^{L_{i}}+\tau_{j}^{R_{i}}\right), j=i, i+1$ and $t=\left(x-x_{i}\right) / h_{i}$, $h_{i}=x_{i+1}-x_{i}$. The square parentheses denote the usual first and second divided differences of the function $S$ with respect to the argument values $x_{j}-\tau_{j}^{L_{i}}, x_{j}, x_{j}+\tau_{j}^{R_{i}}, j=i, i+1$.

Definition 1 A discrete generalized spline is a function $S \in S_{4}^{D G}$ such that

1. for any $x \in\left[x_{i}, x_{i+1}\right], i=0, \ldots, N-1$

$$
\begin{aligned}
S(x) \equiv S_{i}(x)=[ & \left.S\left(x_{i}\right)-\Phi_{i}\left(x_{i}\right) M_{i}\right](1-t) \\
& +\left[S\left(x_{i+1}\right)-\Psi_{i}\left(x_{i+1}\right) M_{i+1}\right] t \\
& +\Phi_{i}(x) M_{i}+\Psi_{i}(x) M_{i+1},
\end{aligned}
$$

where $M_{j}=D_{i, 2} S_{i}\left(x_{j}\right), j=i, i+1$, and the functions $\Phi_{i}$ and $\Psi_{i}$ are subject to the constraints

$$
\begin{aligned}
\Phi_{i}\left(x_{i+1}-\tau_{i+1}^{L_{i}}\right) & =\Phi_{i}\left(x_{i+1}\right)=\Phi_{i}\left(x_{i+1}+\tau_{i+1}^{R_{i}}\right)=0, \\
\Psi_{i}\left(x_{i}-\tau_{i}^{L_{i}}\right) & =\Psi_{i}\left(x_{i}\right)=\Psi_{i}\left(x_{i}+\tau_{i}^{R_{i}}\right)=0, \\
D_{i, 2} \Phi_{i}\left(x_{i}\right) & =1, \quad D_{i, 2} \Psi_{i}\left(x_{i+1}\right)=1
\end{aligned}
$$


2. S satisfies the following smoothness conditions

$$
\begin{aligned}
S_{i-1}\left(x_{i}\right) & =S_{i}\left(x_{i}\right) \\
D_{i-1,1} S_{i-1}\left(x_{i}\right) & =D_{i, 1} S_{i}\left(x_{i}\right), \quad i=1, \ldots, N-1 . \\
D_{i-1,2} S_{i-1}\left(x_{i}\right) & =D_{i, 2} S_{i}\left(x_{i}\right)
\end{aligned}
$$

This definition generalizes the notion of a discrete polynomial spline in [9] and of a generalized spline in $[5,6]$. The latter one can be obtained by setting $\tau_{j}^{L_{i}}=\tau_{j}^{R_{i}}=0, j=i, i+1$ for all $i$. If $\tau_{i}^{L_{j}}=\tau_{i}^{L}$ and $\tau_{i}^{R_{j}}=\tau_{i}^{R}, j=i-1, i$ then according to smoothness conditions (3) the values of the functions $S_{i-1}$ and $S_{i}$ at the three consecutive points $x_{i}-\tau_{i}^{L}, x_{i}, x_{i}+\tau_{i}^{R}$ coincide. Setting $\tau_{j}^{L_{i}}=\tau_{j}^{R_{i}}=\tau_{i}, j=i, i+1$ we obtain $D_{1, i} S(x)=S\left[x-\tau_{i}, x+\tau_{i}\right]$ and $D_{2, i} S(x)=S\left[x-\tau_{i}, x, x+\tau_{i}\right]$, which is the case discussed in [1].

The functions $\Phi_{i}$ and $\Psi_{i}$ depend on the tension parameters which influence the behaviour of $S$ fundamentally. We call them the defining functions. In practice one takes $\Phi_{i}(x)=\Phi_{i}\left(p_{i}, x\right), \Psi_{i}(x)=\Psi_{i}\left(q_{i}, x\right), 0 \leq p_{i}, q_{i}<\infty$. In the limiting case when $p_{i}, q_{i} \rightarrow \infty$ we require that $\lim _{p_{i} \rightarrow \infty} \Phi_{i}\left(p_{i}, x\right)=0$, $x \in\left(x_{i}, x_{i+1}\right]$ and $\lim _{q_{i} \rightarrow \infty} \Psi_{i}\left(q_{i}, x\right)=0, x \in\left[x_{i}, x_{i+1}\right)$ so that the function $S$ in formula (1) turns into a linear function. Additionally, we require that if $p_{i}=q_{i}=0$ for all $i$, then we get a discrete cubic spline. If $\tau_{i}^{L_{j}}=\tau_{i}^{R_{j}}=\tau_{i}$, $j=i-1, i$ for all $i$ then this spline coincides with a discrete cubic spline of [10]. The case $\tau_{i}=\tau$ for all $i$ was considered in [8]. 


\section{Construction of discrete GB-splines}

Let us construct a basis for the space of discrete generalized splines $S_{4}^{D G}$ by using functions which have local supports of minimum length. Since $\operatorname{dim}\left(S_{4}^{D G}\right)=4 N-3(N-1)=N+3$ we extend the grid $\Delta$ by adding the points $x_{j}, j=-3,-2,-1, N+1, N+2, N+3$, such that $x_{-3}<x_{-2}<x_{-1}<a$, $b<x_{N+1}<x_{N+2}<x_{N+3}$.

We demand that the discrete GB-splines $\mathrm{B}_{i}, i=-3, \ldots, N-1$ have the properties

$$
\begin{aligned}
\mathrm{B}_{i}(x) & >0, \quad x \in\left(x_{i}+\tau_{i}^{R_{i}}, x_{i+4}-\tau_{i+4}^{L_{i+3}}\right), \\
\mathrm{B}_{i}(x) & \equiv 0, \quad x \notin\left(x_{i}, x_{i+4}\right), \\
\sum_{j=-3}^{N-1} \mathrm{~B}_{j}(x) & \equiv 1, \quad x \in[a, b] .
\end{aligned}
$$

According to (1), on the interval $\left[x_{j}, x_{j+1}\right], j=i, \ldots, i+3$, the discrete GB-spline $\mathrm{B}_{i}$ has the form

$$
\mathrm{B}_{i}(x) \equiv \mathrm{B}_{i, j}(x)=P_{i, j}(x)+\Phi_{j}(x) M_{j, \mathrm{~B}_{i}}+\Psi_{j}(x) M_{j+1, \mathrm{~B}_{i}},
$$

where $P_{i, j}$ is a polynomial of the first degree and $M_{l, \mathrm{~B}_{i}}=D_{j, 2} \mathrm{~B}_{i}\left(x_{l}\right), l=$ $j, j+1$ are constants to be determined. The smoothness conditions (3) together with the constraints (2) give the following relations

$$
\begin{aligned}
P_{i, j}\left(x_{j}\right) & =P_{i, j-1}\left(x_{j}\right)+z_{j} M_{j, \mathrm{~B}_{i}} \\
D_{j, 1} P_{i, j}\left(x_{j}\right) & =D_{j-1,1} P_{i, j-1}\left(x_{j}\right)+c_{j-1,2} M_{j, \mathrm{~B}_{i}}
\end{aligned}
$$


where

$$
\begin{aligned}
z_{j} & \equiv z_{j}\left(x_{j}\right)=\Psi_{j-1}\left(x_{j}\right)-\Phi_{j}\left(x_{j}\right), \\
c_{j-1,2} & =D_{j-1,1} \Psi_{j-1}\left(x_{j}\right)-D_{j, 1} \Phi_{j}\left(x_{j}\right) .
\end{aligned}
$$

Thus in (6)

$$
P_{i, j}(x)=P_{i, j-1}(x)+\left[z_{j}+c_{j-1,2}\left(x-x_{j}\right)\right] M_{j, \mathrm{~B}_{i}} .
$$

By repeated use of this formula we get

$$
P_{i, j}(x)=\sum_{l=i+1}^{j}\left[z_{l}+c_{l-1,2}\left(x-x_{l}\right)\right] M_{l, \mathrm{~B}_{i}}=-\sum_{l=j+1}^{i+3}\left[z_{l}+c_{l-1,2}\left(x-x_{l}\right)\right] M_{l, \mathrm{~B}_{i}} .
$$

As $\mathrm{B}_{i}$ vanishes outside the interval $\left(x_{i}, x_{i+4}\right)$, we have from $(7)$ that $P_{i, j} \equiv 0$ for $j=i, i+3$. In particular, the following identity is valid

$$
\sum_{j=i+1}^{i+3}\left[z_{j}+c_{j-1,2}\left(x-x_{j}\right)\right] M_{j, \mathrm{~B}_{i}} \equiv 0
$$

from which one obtains the equalities

$$
\sum_{j=i+1}^{i+3} c_{j-1,2} y_{j}^{r} M_{j, \mathrm{~B}_{i}}=0, \quad r=0,1, \quad y_{j}=x_{j}-\frac{z_{j}}{c_{j-1,2}} .
$$


Thus the formula for the discrete GB-spline $\mathrm{B}_{i}$ takes the form

$$
\mathrm{B}_{i}(x)= \begin{cases}\Psi_{i}(x) M_{i+1, \mathrm{~B}_{i}}, & x \in\left[x_{i}, x_{i+1}\right), \\ \left(x-y_{i+1}\right) c_{i, 2} M_{i+1, \mathrm{~B}_{i}}+\Phi_{i+1}(x) M_{i+1, \mathrm{~B}_{i}} & \\ +\Psi_{i+1}(x) M_{i+2, \mathrm{~B}_{i}}, & x \in\left[x_{i+1}, x_{i+2}\right), \\ \left(y_{i+3}-x\right) c_{i+2,2} M_{i+3, \mathrm{~B}_{i}}+\Phi_{i+2}(x) M_{i+2, \mathrm{~B}_{i}} & \\ +\Psi_{i+2}(x) M_{i+3, \mathrm{~B}_{i}}, & x \in\left[x_{i+2}, x_{i+3}\right), \\ \Phi_{i+3}(x) M_{i+3, \mathrm{~B}_{i}}, & x \in\left[x_{i+3}, x_{i+4}\right), \\ 0, & \text { otherwise. }\end{cases}
$$

Substituting formula (9) into the normalization condition (5) written for $x \in\left[x_{i}, x_{i+1}\right]$, we obtain

$$
\begin{aligned}
\sum_{j=i-3}^{i} \mathrm{~B}_{j}(x)= & \Phi_{i}(x) \sum_{j=i-3}^{i-1} M_{i, \mathrm{~B}_{j}}+\Psi_{i}(x) \sum_{j=i-2}^{i} M_{i+1, \mathrm{~B}_{j}} \\
& +\left(y_{i+1}-x\right) c_{i, 2} M_{i+1, \mathrm{~B}_{i-2}}+\left(x-y_{i}\right) c_{i-1,2} M_{i, \mathrm{~B}_{i-1}} \equiv 1 .
\end{aligned}
$$

As according to (5)

$$
\sum_{j=i-3}^{i-1} M_{i, \mathrm{~B}_{j}}=\sum_{j=i-2}^{i} M_{i+1, \mathrm{~B}_{j}}=0
$$

the following identity is valid

$$
\left(y_{i+1}-x\right) c_{i, 2} M_{i+1, \mathrm{~B}_{i-2}}+\left(x-y_{i}\right) c_{i-1,2} M_{i, \mathrm{~B}_{i-1}} \equiv 1 .
$$




\section{Properties of discrete GB-splines}

From here one gets the equalities

$$
y_{i+1}^{r} c_{i, 2} M_{i+1, \mathrm{~B}_{i-2}}-y_{i}^{r} c_{i-1,2} M_{i, \mathrm{~B}_{i-1}} \equiv \delta_{1, r}, \quad r=0,1,
$$

where $\delta_{1, r}$ is the Kronecker symbol. Solving this system of equations and using (8) or (10), we obtain

$$
\begin{aligned}
M_{j, \mathrm{~B}_{i}} & =\frac{y_{i+3}-y_{i+1}}{c_{j-1,2} \omega_{i+1}^{\prime}\left(y_{j}\right)}, \quad j=i+1, i+2, i+3, \\
\omega_{i+1}(x) & =\left(x-y_{i+1}\right)\left(x-y_{i+2}\right)\left(x-y_{i+3}\right)
\end{aligned}
$$

or with the notation $c_{j, 3}=y_{j+2}-y_{j+1}, j=i, i+1$,

$$
\begin{aligned}
M_{i+1, \mathrm{~B}_{i}} & =\frac{1}{c_{i, 2} c_{i, 3}} \\
M_{i+2, \mathrm{~B}_{i}} & =-\frac{1}{c_{i+1,2}}\left(\frac{1}{c_{i, 3}}+\frac{1}{c_{i+1,3}}\right), \\
M_{i+3, \mathrm{~B}_{i}} & =\frac{1}{c_{i+2,2} c_{i+1,3}} .
\end{aligned}
$$

\section{Properties of discrete GB-splines}

The functions $\mathrm{B}_{j}, j=-3, \ldots, N-1$ possess many of the properties inherent in usual discrete polynomial B-splines. To provide inequality (4), in what follows we need to impose additional conditions on the functions $\Phi_{j}$ and $\Psi_{j}$. 


\section{Properties of discrete GB-splines}

The proofs of the following four assertions repeat those given in [5].

Lemma 2 If the conditions

$$
\begin{aligned}
& 0<2 h_{j-1}^{-1} \Psi_{j-1}\left(x_{j}\right)<D_{j-1,1} \Psi_{j-1}\left(x_{j}\right), \\
& 0<2 h_{j}^{-1} \Phi_{j}\left(x_{j}\right)<-D_{j, 1} \Phi_{j}\left(x_{j}\right), \quad j=i+1, i+2, i+3
\end{aligned}
$$

are satisfied, then in (11) $c_{j, k}>0, j=i, \ldots, i+4-k ; k=2,3$, and

$$
(-1)^{j-i-1} M_{j, \mathrm{~B}_{i}}>0, \quad j=i+1, i+2, i+3 .
$$

Theorem 3 Let the conditions of Lemma 2 be satisfied, the functions $\Phi_{j}$ and $\Psi_{j}$ be convex and $D_{j, 2} \Phi_{j}$ and $D_{j, 2} \Psi_{j}$ be strictly monotone on the interval $\left[x_{j}, x_{j+1}\right]$ for all $j$. Then the functions $\mathrm{B}_{j}, j=-3, \ldots, N-1$ have the following properties:

1. $\mathrm{B}_{j}(x)>0$ for $x \in\left(x_{j}+\tau_{j}^{R_{j}}, x_{j+4}-\tau_{j+4}^{L_{j+3}}\right)$, and $\mathrm{B}_{j}(x) \equiv 0$ if $x \notin$ $\left(x_{j}, x_{j+4}\right)$;

2. $\mathrm{B}_{j}$ satisfies the smoothness conditions (3);

3. $\sum_{j=-3}^{N-1} y_{j+2}^{r} \mathrm{~B}_{j}(x) \equiv x^{r}, r=0,1$ for $x \in[a, b], \Phi_{j}(x)=c_{j-1,2} c_{j-2,3} \mathrm{~B}_{j-3}(x)$, $\Psi_{j}(x)=c_{j, 2} c_{j, 3} \mathrm{~B}_{j}(x)$ for $x \in\left[x_{j}, x_{j+1}\right], j=0, \ldots, N-1$. 
Lemma 4 The function $\mathrm{B}_{i}$ has support of minimum length.

Theorem 5 The functions $\mathrm{B}_{i}, i=-3, \ldots, N-1$, are linearly independent and form a basis of the space $S_{4}^{D G}$ of discrete generalized splines.

\section{Local approximation by discrete GB-splines}

According to Theorem 5, any discrete generalized spline $S \in S_{4}^{D G}$ can be uniquely written in the form

$$
S(x)=\sum_{j=-3}^{N-1} b_{j} \mathrm{~B}_{j}(x)
$$

for some constant coefficients $b_{j}$.

If the coefficients $b_{j}$ in (14) are known, then by virtue of formula (9) we can write out an expression for the discrete generalized spline $S$ on the interval $\left[x_{i}, x_{i+1}\right]$, which is convenient for calculations,

$$
S(x)=b_{i-2}+b_{i-1}^{(1)}\left(x-y_{i}\right)+b_{i-1}^{(2)} \Phi_{i}(x)+b_{i}^{(2)} \Psi_{i}(x),
$$

where

$$
b_{k}^{(k)}=\frac{b_{j}^{(k-1)}-b_{j-1}^{(k-1)}}{c_{j, 4-k}}, \quad k=1,2 ; \quad b_{j}^{(0)}=b_{j} .
$$




\section{Local approximation by discrete GB-splines}

The representations (14) and (15) allow us to find a simple and effective way to approximate a given continuous function $f$ from its samples.

Theorem 6 Let a continuous function $f$ be given by its samples $f\left(y_{j}\right), j=$ $-1, \ldots, N+1$. Then for $b_{j}=f\left(y_{j+2}\right), j=-3, \ldots, N-1$, formula (14) is exact for polynomials of the first degree and provides a formula for local approximation.

Proof: It suffices to prove that the identities

$$
\sum_{j=-3}^{N-1} y_{j+2}^{r} \mathrm{~B}_{j}(x) \equiv x^{r}, \quad r=0,1
$$

hold for $x \in[a, b]$. Using formula (15) with the coefficients $b_{j-2}=1$ and $b_{j-2}=y_{j}, j=i-1, i, i+1, i+2$, for an arbitrary interval $\left[x_{i}, x_{i+1}\right]$, we find that identities (17) hold.

For $b_{j-2}=f\left(y_{j}\right)$, formula $(15)$ can be rewritten as

$$
\begin{aligned}
S(x)= & f\left(y_{i}\right)+f\left[y_{i}, y_{i+1}\right]\left(x-y_{i}\right)+\left(y_{i+1}-y_{i-1}\right) f\left[y_{i-1}, y_{i}, y_{i+1}\right] c_{i-1,2}^{-1} \Phi_{i}(x) \\
& +\left(y_{i+2}-y_{i}\right) f\left[y_{i}, y_{i+1}, y_{i+2}\right] c_{i, 2}^{-1} \Psi_{i}(x), \quad x \in\left[x_{i}, x_{i+1}\right] .
\end{aligned}
$$

This is the formula of local approximation. The theorem is thus proved. 
Corollary 7 Let a continuous function $f$ be given by its samples $f_{j}=f\left(x_{j}\right)$, $j=-2, \ldots, N+2$. Then by setting

$$
b_{j-2}=f_{j}-\frac{1}{c_{j-1,2}}\left(\Psi_{j-1}\left(x_{j}\right) f\left[x_{j}, x_{j+1}\right]-\Phi_{j}\left(x_{j}\right) f\left[x_{j-1}, x_{j}\right]\right)
$$

in (14), we obtain a formula of three-point local approximation, which is exact for polynomials of the first degree.

Proof: To prove the corollary, it is sufficient to take the monomials 1 and $x$ as $f$. Then according to (18), we obtain $b_{j-2}=1$ and $b_{j-2}=y_{j}$ and it only remains to make use of identities (17). This proves the corollary.

Equation (15) permits us to write the coefficients of the spline $S$ in its representation (14) of the form

$$
b_{j-2}= \begin{cases}S\left(y_{j}\right)-D_{j-1,2} S\left(x_{j-1}\right) \Phi_{j-1}\left(y_{j}\right)-D_{j, 2} S\left(x_{j}\right) \Psi_{j-1}\left(y_{j}\right), & y_{j}<x_{j}, \\ S\left(y_{j}\right)-D_{j, 2} S\left(x_{j}\right) \Phi_{j}\left(y_{j}\right)-D_{j+1,2} S\left(x_{j+1}\right) \Psi_{j}\left(y_{j}\right), & y_{j} \geq x_{j} .\end{cases}
$$

According to this formula we have $b_{j-2}=S\left(y_{j}\right)+O\left(\bar{h}_{j}^{2}\right), \bar{h}_{j}=\max \left(h_{j-1}, h_{j}\right)$. Hence it follows that the control polygon (e.g., see [4]) converges quadratically to the function $f$ when $b_{j-2}=f\left(y_{j}\right)$, or if the formula (18) is used. 


\section{Recurrence formulae for discrete GB-splines}

Let us define functions

$$
\mathrm{B}_{j, 2}(x)= \begin{cases}D_{j, 2} \Psi_{j}(x), & x \in\left[x_{j}, x_{j+1}\right), \\ D_{j+1,2} \Phi_{j+1}(x), & x \in\left[x_{j+1}, x_{j+2}\right], \quad j=i, i+1, i+2 . \\ 0, & \text { otherwise, }\end{cases}
$$

We assume that the functions $D_{j, 2} \Psi_{j}$ and $D_{j+1,2} \Phi_{j+1}$ are strictly monotone on $\left[x_{j}, x_{j+1}\right)$ and $\left[x_{j+1}, x_{j+2}\right]$ respectively. The splines $\mathrm{B}_{j, 2}$ are a generalization of the "hat-functions" for polynomial B-splines. They are nonnegative and, furthermore, $\mathrm{B}_{j, 2}\left(x_{j+l}\right)=\delta_{1, l}, l=0,1,2$.

According to (9), (11) and (19) the function $D_{2} \mathrm{~B}_{i}$ can be written as

$$
\begin{aligned}
& D_{2} \mathrm{~B}_{i}(x)=\sum_{j=i+1}^{i+3} M_{j, \mathrm{~B}_{i}} \mathrm{~B}_{j-1,2}(x) \\
& =\frac{1}{c_{i, 3}}\left(\frac{\mathrm{B}_{i, 2}(x)}{c_{i, 2}}-\frac{\mathrm{B}_{i+1,2}(x)}{c_{i+1,2}}\right)-\frac{1}{c_{i+1,3}}\left(\frac{\mathrm{B}_{i+1,2}(x)}{c_{i+1,2}}-\frac{\mathrm{B}_{i+2,2}(x)}{c_{i+2,2}}\right) .
\end{aligned}
$$

The function $D_{1} \mathrm{~B}_{i}$ satisfies the relation

$$
D_{1} \mathrm{~B}_{i}(x)=\frac{\mathrm{B}_{i, 3}(x)}{c_{i, 3}}-\frac{\mathrm{B}_{i+1,3}(x)}{c_{i+1,3}},
$$


where

$$
\mathrm{B}_{j, 3}(x)= \begin{cases}\frac{D_{j, 1} \Psi_{j}(x)}{c_{j, 2}}, & x \in\left[x_{j}, x_{j+1}\right), \\ 1+\frac{D_{j+1,1} \Phi_{j+1}(x)}{c_{j, 2}}-\frac{D_{j+1,1} \Psi_{j+1}(x)}{c_{j+1,2}}, & x \in\left[x_{j+1}, x_{j+2}\right), \\ -\frac{D_{j+2,1} \Phi_{j+2}(x)}{c_{j+1,2}}, & x \in\left[x_{j+2}, x_{j+3}\right), \\ 0, & \text { otherwise. }\end{cases}
$$

Using formula (22) it is easy to show that functions $\mathrm{B}_{j, 3}, j=-2, \ldots, N-1$ satisfy the first and second smoothness conditions in (3), have supports of minimum length, are linearly independent and form a partition of unity,

$$
\sum_{j=1}^{N-1} \mathrm{~B}_{j, 3}(x) \equiv 1, \quad x \in[a, b] .
$$

Applying formulae (20) and (21) to the representation (14) we also obtain

$$
D_{1} S(x)=\sum_{j=-2}^{N-1} b_{j}^{(1)} \mathrm{B}_{j, 3}(x), \quad D_{2} S(x)=\sum_{j=-1}^{N-1} b_{j}^{(2)} \mathrm{B}_{j, 2}(x),
$$

where $b_{j}^{(k)}, k=1,2$ are defined in (16). 


\section{Series of discrete GB-splines (uniform case)}

Let us suppose that each step size $h_{i}=x_{i+1}-x_{i}$ of the mesh $\Delta: a=x_{0}<$ $x_{1}<\cdots<x_{N}=b$ is an integer multiple of the same tabulation step, $\tau$, of some detailed uniform refinement on $[a, b]$.

For $\theta \in \mathbb{R}, \tau>0$ define

$$
\mathbb{R}_{\theta \tau}=\{\theta+i \tau \mid i \text { is an integer }\}
$$

and let $\mathbb{R}_{\theta 0}=\mathbb{R}$. For any $a, b \in \mathbb{R}$ and $\tau>0$ let

$$
[a, b]_{\tau}=[a, b] \cap \mathbb{R}_{a \tau} .
$$

The functions $\mathrm{B}_{j, 2}, \mathrm{~B}_{j, 3}$, and $\mathrm{B}_{j}$ with $\tau_{j}^{L_{i}}=\tau_{j}^{R_{i}}=\tau, j=i, i+1$ for all $i$ are nonnegative on the discrete interval $[a, b]_{\tau}$. This permits us to reprove the main results for discrete polynomial splines of [9] for series of discrete generalized splines. Even more, one can obtain the results of generalized splines of [5] from the corresponding statements for discrete generalized splines as a limiting case when $\tau \rightarrow 0$.

In particular, if in (14) and (23) we have the coefficients $b_{j}^{(k)}>0, k=$ $0,1,2, j=-3+k, \ldots, N-1$, then the spline $S$ will be a positive, monotonically increasing and convex function on $[a, b]_{\tau}$.

Let $f$ be a function defined on the discrete set $[a, b]_{\tau}$. We say that $f$ has 
a zero at the point $x \in[a, b]_{\tau}$ provided

$$
f(x)=0 \quad \text { or } \quad f(x-\tau) \cdot f(x)<0 .
$$

When $f$ vanishes at a set of consecutive points of $[a, b]_{\tau}$, say $f$ is 0 at $x, \ldots, x+(r-1) \tau$, but $f(x-\tau) \cdot f(x+r \tau) \neq 0$, then we call the set $X=\{x, x+\tau, \ldots, x+(r-1) \tau\}$ a multiple zero of $f$, and we define its multiplicity by

$$
Z_{X}(f)= \begin{cases}r, & \text { if } f(x-\tau) \cdot f(x+r \tau)<0 \text { and } r \text { is odd } \\ r, & \text { if } f(x-\tau) \cdot f(x+r \tau)>0 \text { and } r \text { is even } \\ r+1, & \text { otherwise }\end{cases}
$$

This definition assures that $f$ changes sign at a zero if and only if the zero is of odd multiplicity.

Let $Z_{[a, b]_{\tau}}(f)$ be the number of zeros of a function $f$ on the discrete set $[a, b]_{\tau}$, counted according to their multiplicity. Let us denote $D_{1}^{L} S(x)=$ $S[x-\tau, x]$.

Theorem 8 (Rolle's Theorem For Discrete Generalized Splines.) For any $S \in S_{4}^{D G}$,

$$
Z_{[a, b]_{\tau}}\left(D_{1}^{L} S\right) \geq Z_{[a, b]_{\tau}}(S)-1
$$


Proof: First, if $S$ has a $z$-tuple zero on the set $X=\{x, \ldots, x+(r-1) \tau\}$, it follows that $D_{1}^{L} S$ has a $(z-1)$-tuple zero on the set $X^{\prime}=\{x+\tau, \ldots, x+$ $(r-1) \tau\}$. Now if $X^{1}$ and $X^{2}$ are two consecutive zero sets of $S$, then it is trivially true that $D_{1}^{L} S$ must have a sign change at some point between $X^{1}$ and $X^{2}$. Counting all of these zeros, we arrive at the assertion (24). This completes the proof.

Lemma 9 Let the function $D_{i, 2} \Phi_{i}$ and $D_{i, 2} \Psi_{i}$ be strictly monotone on the interval $\left[x_{i}, x_{i+1}\right]$ for all $i$. Then for every $S \in S_{4}^{D G}$ which is not identically zero on any interval $\left[x_{i}, x_{i+1}\right]_{\tau}, i=0, \ldots, N-1$,

$$
Z_{[a, b]_{\tau}}(S) \leq N+2 .
$$

Proof: According to (19) and (23), the function $D_{2} S$ has no more than one zero on $\left[x_{i}, x_{i+1}\right]$, because the functions $D_{2} \Phi_{i}$ and $D_{2} \Psi_{i}$ are strictly monotone and nonnegative on this interval. Hence $Z_{\left[a, b_{\tau}\right.}\left(D_{2} S\right) \leq N$. Then according to the Rolle's Theorem 8, we find $Z_{[a, b]_{\tau}}(S) \leq N+2$. This completes the proof.

Denote by $\operatorname{supp}_{\tau} \mathrm{B}_{i}=\left\{x \in \mathbb{R}_{a, \tau} \mid \mathrm{B}_{i}(x)>0\right\}$ the discrete support of the spline $\mathrm{B}_{i}$, i.e. the discrete set $\left(x_{i}+\tau, x_{i+4}-\tau\right)_{\tau}$.

Theorem 10 Assume that $\zeta_{-3}<\zeta_{-2}<\cdots<\zeta_{N-1}$ are prescribed points on the discrete line $\mathbb{R}_{a, \tau}$. Then

$$
D=\operatorname{det}\left(\mathrm{B}_{i}\left(\zeta_{j}\right)\right) \geq 0, \quad i, j=-3, \ldots, N-1
$$


and strict positivity holds if and only if

$$
\zeta_{i} \in \operatorname{supp}_{\tau} \mathrm{B}_{i}, \quad i=-3, \ldots, N-1 .
$$

The proof of this theorem is based on Lemma 9 and repeats that of Theorem 8.66 in $[9$, p.355]. The following statements follow immediately from Theorem 10.

Corollary 11 The system of discrete GB-splines $\left\{\mathrm{B}_{j}\right\}, j=-3, \ldots, N-1$, associated with knots on $\mathbb{R}_{a, \tau}$ is a weak Chebyshev system according to the definition given in $\left[9\right.$, p. 36], i.e. for any $\zeta_{-3}<\zeta_{-2}<\cdots<\zeta_{N-1}$ in $\mathbb{R}_{a, \tau}$ we have $D \geq 0$ and $D>0$ if and only if condition (25) is satisfied. In the latter case the discrete generalized spline $S(x)=\sum_{j=-3}^{N-1} b_{j} \mathrm{~B}_{j}(x)$ has no more than $N+2$ zeros.

Corollary 12 If the conditions of Theorem 5 are satisfied, then the solution of the interpolation problem

$$
S\left(\zeta_{i}\right)=f_{i}, \quad i=-3, \ldots, N-1, \quad f_{i} \in \mathbb{R}
$$

exists and is unique.

Let $A=\left\{a_{i j}\right\}, i=1, \ldots, m, j=1, \ldots, n$, be a rectangular $m \times n$ matrix with $m \leq n$. The matrix $A$ is said to be totally nonnegative (totally 
positive) (e.g., see [3]) if the minors of all order of the matrix are nonnegative (positive), i.e. for all $1 \leq p \leq m$ we have

$$
\operatorname{det}\left(a_{i_{k} j_{l}}\right) \geq 0(>0) \quad \text { for all } \quad \begin{aligned}
& 1 \leq i_{1}<\cdots<i_{p} \leq m, \\
& 1 \leq j_{1}<\cdots<j_{p} \leq n .
\end{aligned}
$$

Corollary 13 For arbitrary integers $-3 \leq \nu_{-3}<\cdots<\nu_{p-4} \leq N-1$ and $\zeta_{-3}<\zeta_{-2}<\cdots<\zeta_{p-4}$ in $\mathbb{R}_{a, \tau}$ we have

$$
D_{p}=\operatorname{det}\left\{\mathrm{B}_{\nu_{i}}\left(\zeta_{j}\right)\right\} \geq 0, \quad i, j=-3, \ldots, p-4
$$

and strict positivity holds if and only if

$$
\zeta_{i} \in \operatorname{supp}_{\tau} \mathrm{B}_{\nu_{i}}, \quad i=-3, \ldots, p-4
$$

i.e. the matrix $\left\{\mathrm{B}_{j}\left(\zeta_{i}\right)\right\}, i, j=-3, \ldots, N-1$ is totally nonnegative.

The last statement is proved by induction based on Theorem 5 and the recurrence relations for the minors of the matrix $\left\{\mathrm{B}_{j}\left(\zeta_{i}\right)\right\}$. The proof does not differ from that of Theorem 8.67 described by $[9$, p.356].

Since the supports of discrete GB-splines are finite, the matrix of system (26) is banded and has seven nonzero diagonals in general. The matrix is tridiagonal if $\zeta_{i}=x_{i+2}, i=-3, \ldots, N-1$. 
An important particular case of the problem, in which $S^{\prime}\left(x_{i}\right)=f_{i}^{\prime}, i=$ $0, N$, can be obtained by passing to the limit as $\zeta_{-3} \rightarrow \zeta_{-2}, \zeta_{N-1} \rightarrow \zeta_{N-2}$.

De Boor and Pinkus [2] proved that linear systems with totally nonnegative matrices can be solved by Gaussian elimination without choosing a pivot element. Thus, the system (26) can be solved effectively by the conventional Gauss method.

Denote by $S^{-}(\mathbf{v})$ the number of sign changes (variations) in the sequence of components of the vector $\mathbf{v}=\left(v_{1}, \cdots, v_{n}\right)$, with zeros being neglected. Karlin [3] showed that if a matrix $A$ is totally nonnegative then it decreases the variation, i.e.

$$
S^{-}(A \mathbf{v}) \leq S^{-}(\mathbf{v}) .
$$

By virtue of Corollary 4, the totally nonnegative matrix $\left\{\mathrm{B}_{j}\left(\zeta_{i}\right)\right\}, i, j=$ $-3, \ldots, N-1$, formed by discrete GB-splines decreases the variation.

For a bounded real function $f$, let $S^{-}(f)$ be the number of sign changes of the function $f$ on the real axis $\mathbb{R}$, without taking into account the zeros

$$
S^{-}(f)=\sup _{n} S^{-}\left[f\left(\zeta_{1}\right), \ldots, f\left(\zeta_{n}\right)\right], \quad \zeta_{1}<\zeta_{2}<\cdots<\zeta_{n} .
$$

Theorem 14 The discrete generalized spline $S(x)=\sum_{j=-3}^{N-1} b_{j} \mathrm{~B}_{j}(x)$ is a variation diminishing function, i.e. the number of sign changes of $S$ does 
not exceed that in the sequence of its coefficients:

$$
S^{-}\left(\sum_{j=-3}^{N-1} b_{j} \mathrm{~B}_{j}\right) \leq S^{-}(\mathbf{b}), \quad \mathbf{b}=\left(b_{-3}, \ldots, b_{N-1}\right) .
$$

The proof of this statement does not differ from that of Theorem 8.68 for discrete polynomial B-splines in [9, p.356].

Acknowledgements: The author was supported by the Thailand Research Fund under grant number BRG/08/2543. He also wishes to express his gratitude to the referees for their invaluable remarks and suggestions for improvements.

\section{References}

[1] P. Costantini, B. I. Kvasov, and C. Manni. On discrete hyperbolic tension splines. Advances in Computational Mathematics, 11:331-354, 1999. C878, C881

[2] C. De Boor and A. Pinkus. Backward error analysis for totally positive linear systems. Numer. Math., 27:485-490, 1977. C897

[3] S. Karlin. Total Positivity, Volume 1. Stanford University Press, Stanford, 1968. C896, C897 
[4] P. E. Koch and T. Lyche. Exponential B-splines in tension. In C.K. Chui, L.L. Schumaker, and J.D. Ward, editors, Approximation Theory VI: Proceedings of the Sixth International Symposium on Approximation Theory. Vol. II, pages 361-364, 1989. Academic Press. C889

[5] B. I. Kvasov. Local bases for generalized cubic splines. Russ. J. Numer. Anal. Math. Modelling, 10:49-80, 1995. C881, C886, C892

[6] B. I. Kvasov. GB-splines and their properties. Annals of Numerical Mathematics, 3:139-149, 1996. C881

[7] B. I. Kvasov. Algorithms for shape preserving local approximation with automatic selection of tension parameters. Computer Aided Geometric Design, 17:17-37, 2000. C878

[8] T. Lyche. Discrete polynomial spline approximation methods. PhD thesis, University of Texas, Austin, 1975. For a summary, see Spline Functions, K. Böhmer, G. Meinardus, and W. Schimpp, editors, Karlsruhe 1975, Lectures Notes in Mathematics No. 501, pages 144-176, Springer-Verlag, Berlin, 1976. C881

[9] L. L. Schumaker. Spline Functions: Basic Theory. John Wiley \& Sons, New York, 1981. C881, C892, C895, C895, C896, C898

[10] Yu. S. Zav'yalov, B. I. Kvasov, and V. L. Miroshnichenko. Methods of Spline Functions. Nauka, Moscow (in Russian), 1980. C881 